\title{
TRAINING AND COMPETENCE
}

\section{Clinical competence in electrophysiological techniques}

Ronald W F Campbell, Richard Charles, J Campbell Cowan, Cliff Garratt, Janet M McComb, John Morgan, Edward Rowland, Richard Sutton

University of

Newcastle upon Tyne and Freeman Hospital, Newcastle upon Tyne, UK

R W F Campbell

The Cardiothoracic Centre-Liverpool, Thomas Drive, Liverpool, UK $\mathrm{R}$ Charles,

The General Infirmary at Leeds, Great George Street, Leeds, West Yorkshire, UK

J C Cowan

Academic Cardiology, Glenfield Hospital, Groby Road, Leicester, UK

C Garratt

Department of Cardiology, Freeman Hospital, Newcastle upon Tyne, UK

$\mathrm{J} M \mathrm{McComb}$

Wessex Cardiothoracic Centre, Southampton, UK

J Morgan

\section{Department of} Cardiological

Sciences, The Medical School, St George's Hospital, London, UK E Rowland

Royal Brompton Hospital, London, UK R Sutton

Correspondence to: Dr Campbell, Professor of Cardiology, University of Newcastle upon Tyne and Freeman Hospital, High Heaton, Newcastle upon Tyne NE7 7DN, UK.

Accepted for publication 7 July 1997
All aspects of health care are under intense scrutiny, none more so than the competence of health care professionals to diagnose and manage disease. Correctly, patients expect those who minister to them to be competent and properly trained. Concepts of what constitutes a good and a bad doctor vary widely. Good may equate with sympathy, yet sympathy is no substitute for ability. The question of competence has received relatively scant attention but is now an important issue. Establishing competence requires setting standards and usually setting conditions for maintaining clinical acumen and ability. Such is laudable, particularly as it can bring about constructive retraining and can divert specific clinical problems to those best able to deal with them.

Specific circumscribed procedures have been an obvious first target for criteria of competence. Such procedures are amenable to audit and are performed in sufficient numbers to establish average rates for success, failure, and efficiency. Standards of competence have been suggested for areas such as angioplasty and stenting. ${ }^{1}$ Cardiac electrophysiological procedures have also been examined and are similarly amenable to suggestions for levels of competence. ${ }^{2}{ }^{3}$ Competence involves exposure to and performance of procedures but more importantly it should include an audit of complications and success. It is to be expected that those with the greatest exposure are likely to be those most competent although this relation is not necessarily so simple. Experience in dealing with complications or anticipating their occurrence plays a large part in the successful performance of many clinical procedures, cardiac electrophysiological ones included.

Establishing and maintaining levels of competence in those performing procedures is very desirable. It identifies those who can be expected to offer safe modern care, and it helps to identify manpower needs for given disease situations.

There are, however, problems with establishing strict guidelines for competence. How can new procedures be incorporated? Radiofrequency ablation is a recently introduced procedure that has taken arrhythmia management by storm. Its safe expansion to the many hundreds of centres worldwide now offering the technique would have been seriously impeded were there competence guidelines in place some five years ago.

In most circumstances competence is based on experience and experience is based on numbers. Selecting appropriate levels of exposure to procedures has attractions but for the most part such numbers are not derived objectively. Is it sufficient to be involved with 50 procedures per year? Seventy five might seem better but that might be an impossible number for all but a few very large centres. Can levels of competence be established for rare diseases? For instance, some types of sophisticated pacemaker and implantable cardioverter-defibrillators may have relatively rare indications ensuring that no single physician can have substantial experience. A similar problem arises with radiofrequency ablation of ventricular tachycardia. Is it enough that practitioners are competent in radiofrequency ablation or must they achieve a specific competence in ablation of each type of arrhythmia? Competence guidelines inevitably disadvantage smaller units where patient turnover is lower. This runs counter to current health policy of decentralising health care yet it may be that in terms of clinical outcome such a move is appropriate.

A further problem is the separation of initial training from continued competence. Training guidelines generally are less contentious than rules that are the basis for continued competence. Are skills really lost quickly? All too often, guidelines suggest levels of continued activity that may be difficult to achieve and that may disadvantage individuals quite capable of safely and successfully undertaking clinical procedures.

This document is an attempt to examine the problems of establishing competence and facilities for the performance of cardiac electrophysiological procedures. It is based on common sense as there are almost no hard data in this field. The one variable that is crucial but cannot easily be catered for is the individual practitioner. Some, with minimal refreshing, may be quite capable of undertaking procedures competently while, for others, consistent high levels of involvement are necessary to maintain skills. Such personal variations are beyond the scope of this document but should be addressed at the level of local audit. 


\section{Cardiac pacing}

Cardiac pacing is a well established discipline involving investigation of patients with bradycardia related symptoms such as syncope, dizziness, dyspnoea, and tiredness. Management involves selection of suitable pacing modes, implantation of devices, and follow up. It is a combined surgical and medical subspeciality. The British Cardiac Society in 1994 recommended an implant rate of 300 new pacing systems per million of the population per year. ${ }^{4}$ That, with the additional replacement of old systems, should translate into 60 procedures per year in the average district general hospital.

INVESTIGATION

Patients presenting with bradycardia related symptoms undergo standard assessment which includes history, physical examination, and 12 lead ECG. Some have more specific tests such as carotid sinus massage, tilt testing, 24 hour ECG, long term ECG recording and, in a few, a bradycardia orientated electrophysiological study is indicated. The selection and order in which these tests are performed for each patient depends on the presentation.

MODE SELECTION

A pacemaker implanter should have personal familiarity with these investigations and be able to interpret the results. These data are the basis of making a decision to pace and are crucial to the selection of optimal pacing mode.

IMPLANTATION

This is a technique requiring some basic training in surgery. The operator should be capable of dissecting the cephalic and subclavian venous system and of percutaneous puncture of the subclavian vein, preferably by both infraand supraclavicular routes, and the internal jugular vein. The implanter should be aware that generators may be implanted deep to pectoralis major but they should not undertake such generator placements unless they are fully familiar with the technique. Usually, the assistance of a trained surgeon should be obtained when deep or unusual implantation sites are to be used. Knowledge of testing of the lead parameters for future sensing and pacing is essential. This includes a basic understanding of the electrical theory underlying pacing function and an ability to use the pacemaker system analyser. ${ }^{5}$ Thus, the operator should be able to perform both the common surgical and the technical aspects of the procedure.

GENERATOR CHANGES

Operators must be familiar with the special problems likely to be encountered at the time of generator change especially the risk of damage to leads. Furthermore, it is necessary that the implanter knows what are acceptable parameters for connection of a new generator to old pacing leads.

IMPLANTATION COMPETENCE-TRAINING

To be considered trained in this field, the Guidelines for Specialist Training in Cardiology ${ }^{6}$ have suggested a need for 75 implants as first operator in the first five years of training with at least $30 \%$ being dual chamber systems. In the sixth year, for the cardiologist making pacing a subspeciality, 70 further implants are recommended with 30 being dual chamber. This makes a total of 145 implants (at least 55 dual chamber) over six years or approximately 24 per year. A lower total of 100 implants, of which at least 40 should be dual chamber, is now recommended for a cardiologist who will practice pacing in a district general hospital.

\section{IMPLANTATION COMPETENCE-MAINTAINING} SKILLS

The training requirements recommended above should give sufficient experience to allow maintenance of skills in both single and dual chamber pacing with annual implantation rates as low as 12 per year. ${ }^{7-9}$ This level should, however, be considered a minimum experience.

COMPLICATIONS OF CARDIAC PACING

The management of severe complications of pacing will remain to some extent the province of the superspecialist. Any averaged sized unit with two implanters performing 25 or more procedures per year will not encounter a need for radical surgical management of infection, lead extraction more than three months after implant, cosmetic pacemaker implantation or femoral implantation more than once or twice per year. Additionally, lead extraction should be performed only in a centre that has immediate access to cardiac surgery.

FOLLOW UP

It is essential that a trainee spends time in the pacemaker clinic as indicated by the guidelines. ${ }^{6}$ Such attendance is equally important for continuing competence. Pacemaker clinics can be technician led provided that clear guidelines are set and patients are being seen on a regular basis by their general practitioner, physician or cardiologist. To maintain and update skills, a pacemaker cardiologist should personally see a substantial number of patients per year with access at the time not only to the pacemaker's programmer but also to echocardiography, exercise stress testing, and long term ECG monitoring.

\section{New pacing centres}

Permanent pacemaker implantation and follow up is conducted both in regional centres and in district hospitals. In 1987 a British Cardiac Society report on cardiology in district hospitals ${ }^{10}$ endorsed the view that permanent cardiac pacing could be carried out safely and effectively in district general hospitals, a view reinforced in the recent second report. ${ }^{11} \mathrm{By}$ 1992 almost one third of all such hospitals provided a permanent pacing service and new centres that continue to be established are almost exclusively in the district hospital setting. In 1995 the national database of the British Pacing and Electrophysiology Group recorded the existence of 135 pacemaker implanting centres (National Pacemaker Database, 1995). The increase in the number of 
implanting centres has been accompanied by a steady improvement in the inappropriately low pacemaker implant rate in the United Kingdom towards the recommended normative need of 300 new implants per million population per year ${ }^{11}$ (in 1994 the implant rate was 258).

While the benefits of devolving permanent pacing from a relatively small number of tertiary centres to a large number of district hospitals have been emphasised by the British Cardiac Society ${ }^{11}$ and by district cardiologists, ${ }^{12}$ the establishment of the new centres has been geographically haphazard. There has been no serious attempt to specify standards of facilities and personnel needed for accreditation of a potential new centre as suitable to provide a pacing service. To date, the only essential ingredients are an enthusiastic physician and a willing purchaser.

To maintain standards in UK cardiac pacing practice, a consensus is required on core criteria that must be met to establish a new centre. They will include geographical justification, personnel, implantation facilities, service and training standards, and a framework for audit of activity and complications.

ADVANTAGES AND DISADVANTAGES OF LOCAL SERVICE PROVISION

The advantages of a local service include clinical, social, and economic factors, ${ }^{12}$ especially if there is no tertiary centre or existing pacing service nearby. Avoidance of travel to a remote hospital (delays in transfer sometimes involve additional risks of infection if a temporary pacing lead is in place ${ }^{13}$ ), and exposure of unmet demand in the local population confer clear advantages. The possible disadvantages of encouraging unrestrained devolution of pacing must also be considered. It is axiomatic that the quality of outcome is related to the institutional patient volume for that procedure, although factors such as case mix are relevant. Parsonnet et al, reported an inappropriately high incidence of pacemaker implant complications when implanters performed fewer than 12 procedures per year. ${ }^{7}$ Conversely, high volume centres achieved low complication rates with no difference between dual and single chamber systems. ${ }^{14}$

Progressive devolution may compromise the ability of existing centres to provide adequate research and training. The appropriateness of pacemaker prescription in new centres may also be an issue. For uncertain reasons, but including inadequate budgets, lack of experience or expertise and competing interests, the National Pacemaker Database documents that new centres, almost exclusively district general hospitals, have a lower rate of implantation of physiological pacing systems than the national average.

Many district hospitals offer high quality pacing services but, as the devolutionary drive continues, it is prudent to question how far the process should go. It would surely be inappropriate for all district hospitals to provide cardiac pacing unless as yet unestablished criteria for the inauguration and operation of a new centre are met.

GEOGRAPHICAL JUSTIFICATION

Populations remote from medical services have a lower take-up rate for those services than populations adjacent to a provider centre. ${ }^{15}$ New pacing centres should be targeted geographically to satisfy unmet need in the local community. Establishment of a new centre adjacent to an established pacing service that is already providing or is capable of providing the volume of service required will merely dilute existing practice with little or no patient benefit, and is unlikely to provide cost effective care.

\section{OPERATIONAL AND EQUIPMENT RESOURCES} Implantation laboratory

The ideal facility for pacemaker implantation is an operating theatre or dedicated pacing laboratory in which the highest standards of sterility can be maintained. While it is both feasible and commonplace to implant pacemakers in cardiac catheterisation laboratories or even in a general radiography department ${ }^{12}$ it is unlikely that operating theatre standards can be maintained in such areas, which should be regarded as suboptimal for pacing.

\section{Equipment}

Relevant equipment includes a pacing systems analyser, an electrocardiographic monitor, a DC defibrillator, an external transthoracic pacemaker, and programmers from each supplier of the generators to be implanted. An adequate toolkit of spare stylets, screwdrivers, repair kits, and patient cables is also necessary. Ideally, the radiolucent operating table should be capable of head down tilt to facilitate percutaneous venous access and to minimise the risk of air embolism. Basic radiographic equipment should incorporate an image intensifier capable of angulated screening as both posteroanterior and oblique-lateral screening are essential for optimal lead placement. The ability to use a stored image is an advantage when contrast injection is required for a difficult venous access. Facilities for computer storage of implant and follow up data are required. Entry of registration data into the National Pacemaker Database is essential and preferably should be by electronic transfer.

\section{Caseload}

An institutional caseload of at least 60 implantations per year should be assured to maintain competence in the centre, although clearly this cannot be achieved within the first year or so of inception. The National Pacemaker Database shows that since 1985, 43 new centres provided a pacing service, 38 of which were district general hospitals. Mean implant rates of six systems in the first year rose to between 20 and 25 systems in the third year, maintained with some variability, at that level by 10 years.

Case mix

A new centre must provide an appropriate mix of pacemaker implants including atrially based 
and rate adaptive pacing. It is thus mandatory that the proposed service be funded to provide an adequate volume of pacing (currently assessed at 300 per million per year) for the target population, and that it includes the costs of complex pacing. The concept that a district centre will intentionally provide predominantly simple (VVI) pacing has always been inappropriate and should now be an anachronism. The National Pacemaker Database records that in 38 new district general hospital centres there was a $12 \%$ rate of DDD implantation in the first year rising to approximately $25 \%$ in the second and subsequent years. Implantation rates for ventricular rate adaptive (VVIR) systems were approximately $8 \%$ throughout.

Personnel

A pacemaker implanting centre should have a cardiologist trained and active in cardiac pacing. A pacemaker service implies a 24 hour a day, year round commitment. A single implanting physician will leave the service uncovered during periods of absence, which is suboptimal; therefore, centres should ensure the provision of at least two trained implanters. When there is only a single implanting physician, a clear protocol for collaboration with the regional centre or an adjacent pacing service is mandatory. A fully trained physiological measurement technician who works regularly in pacing, and access to a bioengineering service for equipment maintenance are required.

Audit

It is incumbent on all implanting centres to collect audit data describing their activity, clinical case mix, pattern of mode prescription, and complication rates. The data should be available to institutional audit meetings, purchasing authorities, and preferably to public inspection allowing informed comparison between centres. Such data provide a strong incentive to optimise local performance both by the clinical service providers who will wish to demonstrate excellence, and by purchasers who will wish to be informed of the adequacy of funding. The publication of national guidelines for appropriate pacemaker prescription allows the setting of agreed standards of service provision. Audit data are also required by the National Pacemaker Database for national and international comparison.

\section{RETRAINING OF PHYSICIANS IN PACEMAKER} IMPLANTATION

New pacemaker implanting services in district hospitals may sometimes be provided and supervised by physicians who were trained in cardiac pacing, but who have not maintained their operative competence and familiarity with new developments in pacing. Clearly, all such physicians must be retrained. The extent and intensity of retraining will vary considerably between individuals. It will depend on the duration of non-involvement with pacing, performance levels of other practical vascular procedures (such as diagnostic cardiac catheterisa- tion), inherent operative dexterity, and ability to take a significant period of study leave.

Prospective previously trained implanting physicians should seek retraining if their practical skills have lapsed for two or more years. Re-accreditation should involve a minimum supervised experience as first operator in an established pacing centre. Competent placement of permanent pacing leads will depend on several factors including the extent of previous training especially in atrial lead placement. Re-accreditation as an implant physician should comprise performance of a minimum of 20 supervised, first operator implants. Where the original training included competence in atrial lead placement at least 10 of the implants should include an atrial lead. If original training covered only ventricular lead placement, at least 15 of the implants should include an atrial lead.

Retraining must involve attendance at the training centre's pacemaker follow up clinic to provide familiarisation with routine evaluation, programming, assessment of rate adaptive pacing systems, and troubleshooting. While the retrained physician will clearly wish to maximise proficiency, purchasers should require evidence of adequate retraining where necessary before purchasing the new pacing service.

\section{Interventional cardiac electrophysiology}

Diagnostic electrophysiology was established in 1969. For most of the time since then, it has been a very specialised subspecialty activity in cardiology. Familiarity with the technique was not a requirement for general cardiological accreditation and the few who entered the field devoted a majority of their time to it. Electrophysiological concepts are complex. To master them demands considerable teaching and experience. The very rapid developments in technology (principally multichannel data recording) and in the understanding of arrhythmia anatomy and physiology has created difficulties for establishing training and continuing competence guidelines.

The advent of ablation as a catheter based treatment for arrhythmias dramatically increased the profile of clinical cardiac electrophysiology and has encouraged more trainees and the establishment of more centres.

It has rapidly become inappropriate to define competence guidelines for radiofrequency ablation separately from diagnostic electrophysiology; both procedures are inextricably interlinked. There is, however, a real danger that an emphasis on ablation may curtail diagnostic procedures and reduce the investigative competence of those who now train in this field.

TRAINING

Published recommendations appear to be only loosely based on facts. The American College of Cardiology (ACC) recommends 12 months' clinical training in electrophysiology and recommends that each trainee should actively participate in 100 procedures, including 50 initial procedures. ${ }^{3}$ Numbers of ablations are not specified. The North American Society for Pacing and Electrophysiology (NASPE) 
recommends that a trained physician, already experienced in electrophysiology, should have been primary operator in 30 ablations, including 15 accessory pathway ablations. ${ }^{6}$ The ACC subsequently reiterated these numbers. ${ }^{2}$ The British Cardiac Society has recommended that cardiac trainees who wish to specialise in electrophysiology participate in five ablations during the first five years of training, and in 70 electrophysiology studies and 50 ablations in the final year. ${ }^{16}$

Therefore, published documents ${ }^{2316}$ in the UK and the US suggest that it may be necessary to have participated in 50 ablations, and have been primary operator in 30 to be considered trained in interventional electrophysiology. No recommendations have been made concerning the success of procedures as a training requirement.

CONTINUING COMPETENCE

NASPE recommends that to maintain competence a physician should undertake 20 ablations per year. The ACC Cardiovascular Technology Assessment Committee has recommended that physicians undertaking catheter ablation should perform at least 20 ablations per year. ${ }^{2}$ This number appears to be based on the NASPE 1992 survey, which has been published only as a summary. ${ }^{6}$ The ACC recommendations probably derive from the fact that low volume centres (fewer than 20 procedures a year) had a higher complication rate $(3.2 \%)$ than did high volume centres (more than 50 procedures per year) (1.5\%). The logical inference from these data might be that ablation should be restricted to centres performing more than 50 rather than more than 20 ablations per year. NASPE recommends that to assure continued competence, success rates of at least $80 \%$ for superventricular tachycardia and at least $30 \%$ for ventricular tachycardia should be achieved. These success rates are empiric. ${ }^{6}$ There are no other data available regarding number of cases required to maintain continuing competence.

\section{LEARNING CURVES}

Limited information is available about either individual operator or institution learning curves. Most, if not all, of this information relates to accessory pathways rather than to $\mathrm{AV}$ nodal modification or other procedures. Schluter et al reported 92 patients with accessory pathways. ${ }^{18}$ Their success rate was $50 \%$ for the first 10 and $100 \%$ for the last 40 ; after patient 52 they were invariably successful. By this stage they were however doing 10 ablations each month.

Leather et al reported an overall success rate of $71 \%$ for ablation of accessory pathways. ${ }^{19}$ The success rates rose over successive three month periods $(52 \%, 60 \%$, and $90 \%)$. This group however were performing large numbers of ablations in a short time. They achieved success rates of $90 \%$ after 46 cases. The learning curve for left free wall pathways was steeper than for pathways in other locations.

Katritsis et al noted significantly decreased procedure and screening times with increasing experience. $^{20}$ Their success rates also rose as experience grew. They considered 71 consecutive cases done by the same first operator, and found that screening time improved by $67 \%$, procedure time by $47 \%$, and number of discharges by $50 \%$ as experience grew. They do not comment on success rates.

Calkins et al showed that for three attending physicians and eight electrophysiology fellows the percentage of their 400 adult patients in whom catheter ablation was initially successful increased with experience, from $76 \%$ in the first 50 patients to $96 \%$ in the eighth group of 50 patients. ${ }^{21}$ A statistically significant increase was seen only after 250 ablations, although after 100 cases the success rate was about $85 \%$. Mean (SD) procedure time decreased from 138 (59) to 79 (41) minutes.

The Pediatric Electrophysiology Society ablation registry ${ }^{22}$ has described in detail the learning curve for radiofrequency ablation in children in the United States. In the first 1546 procedures reported to its registry, success (that is, inability to induce tachycardia at the end of the procedure) was initially $71 \%$. Half the improvement (to $91 \%$ ) occurred after 19 cases, and $90 \%$ of the improvement after 53 cases. There was a steep learning curve for left free wall pathways $(90 \%$ after 26 cases with overall success rates of $94 \%$ being achieved). This learning curve differed from that for right free wall pathways (90\% after 282 cases with overall success rates of $100 \%$ being achieved). Complication rates fell with experience. The initial complication rate was $17 \%$, falling to $6 \%$. The $50 \%$ and $90 \%$ learning milestones occurred at 27 and 90 cases. Procedure time and, perhaps more importantly, fluoroscopy time fell with experience. Procedure time fell from 326 to 247 minutes, with $90 \%$ expected at case 65 . Fluoroscopy time fell from 72 to 36 minutes, with $50 \%$ at 72 cases and $90 \%$ at 240 cases.

\section{INFERENCES FROM LEARNING CURVES}

As experience grows, success increases and complication rates fall. After 50 cases, a $90 \%$ success rate can be expected ${ }^{18} 1922$ (although this will be achieved earlier for left free wall pathways). Ninety per cent of the learning curve, with acceptably low complication rates (90\% of the improvement) is achieved after about 90 cases. ${ }^{22}$ It should be emphasised that this experience of radiofrequency ablation comes from centres and individuals with a long experience (more than 10-15 years) in electrophysiology.

There is no information available about levels of experience required for continuing competence, other than the data from the NASPE survey regarding complications from high and low volume centres ${ }^{26}$ and these may reflect the initial learning curve rather than continuing competence.

The British Cardiac Society recommends that 50 ablations per year are necessary for training. This implies that to be a training centre, 50 ablations plus at least 20 electrophysiological studies per year be done. Although there are no reliable data, it is thought that 
there are only perhaps five centres in the UK achieving this throughput. The scarce published data suggest that, to be a designated training centre, there should be experience of at least 90 accessory pathway ablations, with a minimum success rate of $90 \%$, and a complication rate of no more than $6 \%$. (This complication rate is a substantial overestimate as minor complications such as bruising and temporary bundle branch block were included.) The Multicentre European Radiofrequency Survey (MERFS) reported a complication rate of $5.1 \%$ overall, with a rate of $4.4 \%$ in those undergoing accessory pathway ablation, $8.0 \%$ AV nodal modification, and $3.2 \%$ AV node ablation. ${ }^{23}$ Pooled data from NASPE reveal a complication rate of $2.1 \%$ with $0.2 \%$ mortality in accessory pathway ablation and $0.96 \%$ in AV node ablation, and a rate of AV block in AV nodal modification of $5.7 \%$ (18 of 315 patients). ${ }^{26}$

STAFF

Rosenheck et al have demonstrated that an effective service may be provided by a single experienced physician and a nurse, although details of other staff necessarily involved were not given. ${ }^{24}$ NASPE and the ACC recommend that laboratory personnel should include two nurses or technicians. ${ }^{26}$ Others have recommended that two physicians should be present, although one might be in training. ${ }^{2}{ }^{6}$

\section{Maintenance of competence}

The complexity of electrophysiology interventions requires a minimum number of procedures to be undertaken annually to maintain competence. At present in the UK most operators retain their proficiency as they are either the only electrophysiologist in their unit or, where there are two senior operators, the majority of ablations are performed as a single operator procedure. In most cases the principal operator is assisted by a junior in training. At present this is satisfactory for the great majority of interventional electrophysiological procedures and appears to be associated with a trend towards shortened procedure times and maintenance of high success rates.

The field of interventional electrophysiology is developing rapidly. The expansion of ablation techniques to patients with ventricular tachycardia and atrial arrhythmias (especially atrial flutter and fibrillation) may require that two individuals, both trained to a high level in the interpretation of endocardial electrograms and in the positioning of ablation catheters, be simultaneously involved in single procedures. At the moment single operator UK centres probably offer a safe and effective service, but the caseload is dominated by patients with accessory pathways. If the anticipated expansion of ablation techniques to ventricular tachycardia and atrial fibrillation does occur, such centres will have to increase or redeploy staff to accommodate expansion in the field. For small centres this may affect their viability if there is insufficient caseload or case mix to maintain individual skills.
AUDIT OF SUCCESS RATE OF INTERVENTIONAL PROCEDURES

Unlike many cardiac interventional procedures, the success or failure of radiofrequency ablation is clearly defined. On completion of a procedure, conduction over an accessory pathway is either still present or it has been successfully interrupted. Likewise, an arrhythmia is either inducible or it has been rendered non-inducible. In most radiofrequency ablation procedures therefore, short term outcome can be dichotomised into success or failure, which makes outcome readily amenable to audit.

The purpose of such audit is to enable individual operators and institutions to compare their success rate with that of colleagues in other institutions. Audit can help define desirable and attainable standards. At present, no such standards exist. There are large published series reporting the success rates of ablation from American and European centres. These success rates are in general very high. It is unclear whether these series provide realistic comparisons for unselected patients managed in smaller centres in the UK by cardiologists who in most cases fulfil a more general cardiological role than their European and American counterparts.

The NASPE 1992 survey, published only in summary, showed a $91 \%$ success rate for left free wall pathways, $87 \%$ for septal pathways, and $82 \%$ for right free wall pathways, with significant complications in $2.1 \%$ and deaths in $0.2 \%{ }^{6}$ The success rates for AV nodal modification were $96 \%$ (slow pathway ablation) and $90 \%$ (fast pathway ablation), with AV block in $1 \%$, and no deaths. Success for complete AV nodal ablation was 95\% with two (of 1600) procedure related deaths. The success rate in atrial tachycardia/atrial flutter was $75 \%$ and in ventricular tachycardia $71 \%$ (85\% in patients with structurally normal hearts, $54 \%$ in those with ischaemic heart disease, and $61 \%$ in those with cardiomyopathy). Published requirements for success rates bear little relation to these figures. $^{6}$

It seems desirable that success rates from individual centres and individual operators should be subjected to peer comparison, rather than to comparison with previously published reports. This can readily be achieved if each centre in the UK performing interventional electrophysiology submits data to a central database. Such a database already exists but reporting of data is incomplete. It should be incumbent on any centre performing interventional electrophysiology to submit their data to the national database.

For the present, the success rates of individual centres and operators would remain confidential and would be made available only to the centre or individual concerned. Individual centres would critically assess their success rates in comparison with national figures and determine where any inadequacies might lie. This approach would be in keeping with the approach currently adopted by cardiothoracic surgeons in the UK. 
In determining procedural success rates, case mix is a particularly important consideration. Interruption of $\mathrm{AV}$ nodal conduction in general shows a higher success rate than accessory pathway ablation; pre-excited pathways are easier to treat than concealed pathways and left sided pathways are in general easier to ablate than other locations. Success rates for ablation of intra-atrial arrhythmias and more particularly ventricular tachycardia are significantly lower again. It is therefore of particular importance that sufficient information be compiled to assess success rates in relation to case mix and to identify, for individual operators and centres, where strengths and weaknesses lie.

While audit of short term success of ablation procedures on leaving the catheter laboratory is relatively easy, audit of longer term success is more difficult. It is nonetheless important that data on long term success of ablation should be gathered to enable recurrence rates to be assessed. Long term follow up will therefore need to be an integral part of the audit process.

\section{AUDIT OF ELECTRICAL PROCEDURES BASED ON} COMPLICATIONS

The provision of invasive arrhythmia services at a local level has clear advantages for patients but it is important that this is not at the expense of the standard of care that patients are likely to receive. To some extent it is unavoidable that newly established centres will have a learning curve for radiofrequency catheter ablation success, but it is arguably more important from the patient's point of view that complication rates should be kept to a minimum. Patients undergoing these procedures are usually young adults with a normal or near normal prognosis. Whereas failure of the procedure in a greater than expected number of patients is inconvenient for the patient and may result in referral to a more experienced centre, a serious complication such as tamponade or coronary arterial damage may have catastrophic consequences. The pressure to achieve a very high first procedure success rate that might result from an open audit based on these figures may well be counterproductive, leading to an increase in procedure times and complications.

A voluntary, retrospective survey (MERFS) recorded that complications are highest in AV nodal modification procedures and may reach $8 \%$. There is also a reasonably strong possibility of under reporting with these figures. There is a strong case for open prospective audit based on complication rates in the $\mathrm{UK} .^{23}$

\section{Implantable cardioverter-defibrillators}

The implantable cardioverter-defibrillator (ICD) is a powerful, but expensive, means of controlling malignant ventricular arrhythmias. In contrast to radiofrequency catheter ablation, there are no data regarding ICD implantation learning curves, and no information addressing the amount of training or experience required to select patients for this device. Currently there are no centres in the UK implanting more than 30 devices annually, although this may change dramatically in the next few years depending on the outcome of a number of large randomised trials. For these reasons, it is probably not possible to stipulate training requirements (in terms of specific numbers) for ICD implantation in the $\mathrm{UK}$ at this time. Indeed, with the increasing ease of implantation of the device (now equivalent to a large single chamber pacemaker ${ }^{25}$ ), it may be appropriate to consider other aspects of cardiological training (for example, pacemaker implantation) as a surrogate for particular aspects of ICD management. Implantation of the device is perhaps the least difficult aspect of ICD use however, and it is important that training in diagnostic electrophysiology (see above) is a compulsory component of training in ICD use. In terms of both training and continuing competence for ICDs it is logical to apply the same numbers that have been suggested separately for pacemakers and electrophysiological studies. Programming of ICDs is more complex than for pacemakers, and training and continuing competence in ICD use should include practical instruction and continuing experience in device programming, as well as pacemaker implantation and diagnostic electrophysiology.

It is clear that there are a number of different areas of expertise required in the management of patients who are candidates for ICDs. In many hospitals, all these skills may be embodied in a single cardiologist. This does not necessarily have to be the case, however, and in large centres a team approach is likely to be employed. The different areas of expertise required can be conveniently divided into four groups: diagnosis of ventricular arrhythmias; risk assessment and selection of therapy in patients with these arrhythmias; implantation of the device; and programming and troubleshooting of device function.

\section{DIAGNOSIS OF VENTRICULAR ARRHYTHMIAS}

Clinicians involved in selecting patients for device therapy must be fully trained in diagnostic electrophysiological techniquesthat is, they must have performed and interpreted at least 70 diagnostic studies in their sixth training year. They need be able to recognise and understand the significance of bundle branch reentrant tachycardia, ${ }^{26}$ right ventricular outflow tract tachycardia, ${ }^{27}$ fascicular tachycardia, ${ }^{28}$ and other conditions for which catheter ablation is likely to be the most appropriate therapy. They need to be able to differentiate ventricular tachycardia from antidromic and Mahaim tachycardias, and exclude latent pre-excitation in patients presenting with idiopathic ventricular fibrillation. They should be able to identify patients with scar related postmyocardial infarction ventricular tachycardia who might be appropriate candidates for catheter ablation therapy. ${ }^{29}$ It is not strictly necessary that the physician be skilled or fully trained in catheter ablation techniques to be able select patients for ICD treatment, but he or she must be aware of the indications for this approach. In addition to initial training in electrophysiology, competence should be maintained and for this reason it would be difficult 
to support the development of a defibrillator programme at a hospital in which a diagnostic electrophysiology service were not available.

RISK ASSESSMENT AND SELECTION OF THERAPY The cost of ICDs is such (approximately $£ 27000$ total inhospital cost for implant) that cardiologists selecting patients for this treatment should be able to provide an informed estimate, as far as possible given up to date information, of the likely benefit to the patient in terms of prognosis and symptom control. Risk assessment for patients with ventricular arrhythmias is dependent on performance and interpretation of a number of investigations other than diagnostic electrophysiology: coronary arteriography, ventricular angiography, and echocardiography are required in most cases, therefore, adequate expertise in these areas is important. Informed interpretation of right ventricular angiograms and right ventricular echocardiographic views may be required, and the role of endomyocardial biopsy, magnetic resonance imaging, and signal averaging should be understood. Cardiologists selecting patients for ICD treatment should be able to perform and interpret ventricular stimulation studies with regard to their prognostic value. ${ }^{30} 31$

DEVICE IMPLANTATION AND MANAGEMENT OF PHYSICAL COMPLICATIONS

Many of the components of ICD implantation and management of complications are shared with pacemakers. It would be expected that the standards considered a minimum in terms of training and continuing competence for pacemaker implantation should apply to ICDs. It is necessary, therefore, that the ICD implanter be fully trained in pacemaker implantation (at least 145 implants of which 70 should have been in their sixth training year.) In terms of continuing competence, an ICD implanter should be performing at least 12 pacemaker (see above) or 12 ICD implantations per year to maintain appropriate skills. As with pacemaker implants, specific training in subpectoral implantation is necessary. Training in surgical technique for abdominal implants should not be necessary as long as a surgeon is available on site.

PROGRAMMING AND TROUBLESHOOTING THE DEVICE

Although implantation of an ICD may be as straightforward as that of a single chamber pacemaker, programming the device is considerably more complex. If the device is to be implanted for recurrent ventricular tachycardia (rather than pure ventricular fibrillation), a practical knowledge of the risks and benefits of antitachycardia pacing is required. A number of problems remain to be completely solved, including the occurrence of inappropriate discharges, pain associated with the discharge, and psychological consequences of implantation of the device. The physician should be aware of these and of methods for their prevention and management. Specific supervised ICD programming experience is a necessary part of training, for instance, in the use of the various detection criteria and levels of treatment, and ensuring safety of the device. It would be expected that such experience could be gained during the specialist (sixth) year of the proposed training scheme, but this cannot be assumed even in a UK "arrhythmia centre" performing large numbers of pacemaker implants and radiofrequency catheter ablations. Training should be supplemented by the use of training courses currently run by individual ICD manufacturers. Continuing competence in programming and troubleshooting is likely to be maintained only if patients with ICDs are seen regularly in a follow up defibrillator clinic. It is clear that there is a minimum size of clinic below which such experience cannot be gained, and it is recommended that, once established, at least 15 patients be attending such a clinic.

\section{Paediatric electrophysiological techniques-a special case}

The diagnosis and management of paediatric arrhythmias is a growing and important clinical activity. The range and patterns of arrhythmogenic conditions in children is different from that seen in adult practice. Moreover, the incidence of serious arrhythmia disturbances is much lower, principally as there is no arrhythmic contribution from coronary artery disease. Paediatric electrophysiology, be it diagnostic invasive electrophysiology, radiofrequency ablation, pacing, or ICD implantation, is a specialised low volume activity. It is impossible that those working in this field could attain the experience levels for training and continuing competence described in the preceding sections if their experience were to be based on paediatric procedures alone. It is recommended that paediatric electrophysiological specialists undertake the same numbers of training procedures as suggested for their adult counterparts but that up to $75 \%$ of that experience could be from adult procedures.

For continuing competence we cannot specify figures. It is unreasonable to expect that a paediatric electrophysiologist would perform adult investigations and techniques to maintain competence. As with other low volume specialist procedures, we expect continued competence to be maintained by the physician's total involvement in all the techniques undertaken in their unit. It would be very rare for a paediatric unit to have more than one paediatric electrophysiologist, thus experience will be concentrated on that individual. In the event of a particularly complex procedure being necessary, we recommend that two or more paediatric electrophysiologists collaborate.

\section{Institutional aspects of ablation and ICD therapy}

There are 31 centres in the UK and the Republic of Ireland performing catheter ablation procedures, 34 centres performing ICD implantation, and 135 performing permanent pacemaker implantation. This section will deal with catheter ablation and ICD implantation, and follow up. The number of centres offering these treatments at present is similar, predominantly 
because they are modern treatments for patients with complex arrhythmias. Twenty eight centres perform all three treatments, six undertake pacemaker and ICD implants, and three do pacemaker implants and catheter ablation.

The similarities in technical requirements are few. At present there are no controls to determine which institutions can offer these treatments, although they generally are available at regional centres. The demand for procedures to be offered at local level, or at least to be more widely available than at present, has increased the number of sites at which these procedures are being performed over the last five years.

Both catheter ablation and ICD use are important in the treatment of complex arrhythmias, and both should be offered at centres that provide treatment for patients with these conditions. This concept should be a central tenet of institutional competence-that a certain level of facility should be mandatory at all institutions offering modern interventional treatment for cardiac arrhythmias. This should include but not be limited to: catheter ablation, ICD implantation, insertion of temporary pacemakers, coronary angiography, and echocardiography.

A more contentious issue is whether other techniques for the treatment of complex arrhythmias, such as antitachycardia surgery, should be available on site. At present the estimated number of patients undergoing these additional treatments is very small, probably around 50 per year. It is doubtful whether on this caseload more than a few centres can maintain their expertise. It would therefore seem sensible to designate these treatments as a supraregional service. Familiarity with these techniques and their therapeutic value however, must be maintained by the physicians in centres where these treatments are not available.

FACILITIES AND EQUIPMENT

The requirements for equipment suitable for undertaking routine invasive electrophysiological investigation, such as multichannel physiological recorders and stimulators, has been published elsewhere. Such equipment must be regularly inspected and maintained to stipulated standards in association with the medical physics department or other agencies.

These procedures require $x$ ray screening equipment that in the case of ICD implantation can be relatively simple, but in the case of catheter ablation requires modern state of the art equipment. Catheter ablation procedures may still be lengthy, and digitised fluoroscopy equipment is essential to minimise the dose delivered to the patient and the operator. Rotational $\mathrm{C}$-arm equipment and acquisition and storage of images, as well as the ability to display freeze-frame is essential. Biplane fluoroscopy is desirable but not essential.

Power sources for catheter ablation require regular inspection and maintenance to ensure that they are performing within specified tolerances.
Patients undergoing ICD implantation or catheter ablation often have cardiac abnormalities in addition to their rhythm disorder, or dysfunction may arise as a result of such procedures. A comprehensive range of investigational procedures and interventions should be available for the appropriate investigation of patients in whom these arrhythmia treatments are being considered, and the prompt rescue of those in whom complications occur. It is recommended that these be available at the same site.

\section{MEDICAL, TECHNICAL, AND NURSING STAFFING} LEVELS

Despite the improvements in procedure and screening times that have been achieved for catheter ablation during the past few years, the procedures remain time consuming. Moreover, if as seems probable the technique becomes more widely available for the treatment of atrial and ventricular arrhythmias, there will be a return to longer and more complex procedures. It is recommended that institutions offering complex catheter ablation should have two physicians trained in these procedures. Similar recommendations are proposed for ICD implantation.

Cardiac technicians provide an important and essential role in both procedures. They provide an even more essential role in follow up of patients with ICDs. Appropriate levels of training in these techniques are not a mandatory part of cardiac technician training. At least two appropriately trained staff, who should be MTO grade 3-4 and who should have undertaken a specialist training module or equivalent, should be part of the hospital complement of cardiac technicians. There are no specific requirements for nursing training other than that nurses staffing catheter laboratories and those involved in the ward care of these patients should be familiar with the presentation, management, and complications seen in patients with complex arrhythmias.

1 Parker DJ, Gray HH, Balcon R, et al. Planning for coronary angioplasty: guidelines for training and continuing competence. British Cardiac Society and British Cardiovascular Intervention Society Working Group on interventional cardiology. Heart 1996;75:419-25.

2 American College of Cardiology Cardiovascular Technology Assessment Committee. Catheter ablation for cardiac arrhythmias: clinical applications, personnel and facilities. $\mathcal{F}$ Am Coll Cardiol 1994;24:828-33.

3 Flowers NC, Abildskov JA, Armstrong WF, et al. ACC policy statement. Electrophysiology/Electrocardiography Subcommittee ACC. Recommended guidelines for training in adult clinical cardiac electrophysiology. ACC policy ing in adult clinical cardiac electrophysiology.

4 British Cardiac Society. Council statement on the demand British Cardiac Society. Council statement on the demand
for cardiac services and the development of a waiting list strategy for cardiac disease. http://www.bcs.rbh.nthames. nhs.uk/bcs.nsf, July 1994

5 Sutton R, Bourgeois I. The foundations of cardiac pacing. An illustrated practical guide to basic pacing. New York: Futura Publishing Co, 1991.

6 Hall RJC, Boyle RM, Webb-Peploe M, Chamberlain DA, Parker DJ. Guidelines for specialist training in cardiology. Br Heart $\mathcal{F}$ 1995;73(suppl 1):1-24.

7 Parsonnet V, Bernstein AD, Lindsay B. Pacemaker implantation complication rates: an analysis of some contributing factors. F Am Coll Cardiol 1989;13:917-21.

8 Hall JA, Grace AA, Newell SA, Cole T, Schofield PM, Shapiro LM, et al. Prospective evaluation of the early piro LM, et al. Prospective evaluation of the early maker implantations. European fournal of Cardiac Pacing and Electrophysiology 1993;3:14-20.

9 Hargreaves MR, Doulalas A, Ormerod OJM. Early compliHargreaves MR, Doulalas A, Ormerod OJM. Early compli-
cations following dual chamber implantation: 10 year 
experience of a regional pacing centre. European fournal of Cardiac Pacing and Electrophysiology 1995;5:133-8.

10 A report of a working group of the British Cardiac Society: cardiology in the district hospital. Br Heart f 1987;58:53746.

11 A report of a working group of the British Cardiac Society: cardiology in the district hospital. Br Heart f 1994;72:3038.

12 Pitcher D. Cardiac pacing: the service should be as local as the anaesthetic. Br Heart f 1995;74:7-8.

13 Andrews R, Skehan JD. Temporary pacing: continuing failures in general medical management [abstract]. Br Heart $\mathcal{F}$ 1992;68:91P

14 Aggarwal RK, Connelly DT, Ray SG, Ball J, Charles RG. Early complications of permanent pacemaker implantation: no difference between dual and single chamber systems. Br Heart f 1995;73:571-5.

15 Rickards AF. Where's the block? [editorial]. BMF 1984;288 737-8.

16 Scheinman MM NASPE policy statement: Catheter ablation for cardiac arrhythmias, personnel and facilities. PACE 1992;15:715-21.

17 Scheinman MM. Patterns of catheter ablation practice in the United States. Results of the 1992 NASPE survey [editorial]. PACE 1994;17:873-5.

18 Schluter M, Geiger M, Siebels J, Dulceck W, Kuck KH Catheter ablation using radiofrequency current to cure symptomatic patients with tachyarrhythmias related to an accessory atrioventricular pathway. Circulation 1991;84 $1644-61$

19 Leather RA, Leitch JW, Klein GJ, Guiraudon GM, Yee R, Kim YH. Radiofrequency catheter ablation of accessory 1651-5.

20 Katritsis D, Bashir Y, Heald S, Polonieclci J, Ward DE. Radiofrequency ablation of accessory pathways: implications of accumulated experience and time dedicated to procedures. Eur Heart f 1994;15:339-44.

21 Calkins H, el-Atassi R, Kalbfleisch SJ, Langberg JJ, Morady $\mathrm{F}$. Effect of operator experience on outcome of radiofre-
quency catheter ablation of accessory pathways. Am 7 Carquency catheter ablation
diol 1993;71:1104-5.
22 Danford DA, Kugler JD, Deal B, Case C, Friedman RA, Saul JP, et al. The learning curve for radiofrequency Salation of tachyarrhythmias in pediatric patients. Participating members of the Pediatric Electrophysiology Society. Am f Cardiol 1995;75:587-90.

23 Hindricks G. Complications of radiofrequency catheter ablation of arrhythmias. The Multicentre European Radiofrequency Survey (MERFS) investigators of the Working Group on Arrhythmias of the European Society of Cardiology. Eur Heart F 1993;14:1644-53.

24 Rosenheck S, Sharon Z, Weiss A, Agmon Y, Weiss AT, Gotsman MS. Single physician approach to radiofrequency catheter ablation in patients with supraventricular tachycardia. PACE 1993;16:2112-17.

25 Fitzpatrick AP, Lesh MD, Epstein LM, Lee RJ, Siu A, Merrick S, et al. Electrophysiological laboratory, electrophysiologist-implanted, non-thoracotomyimplantable cardioverter defibrillators. Circulation 1994; 89:2503-8.

26 Tchou P, Jazayeri M, Denker S, Dongas J, Caceres J, Akhtar M. Transcatheter electrical ablation of the right bundle branch: a method of treating macro-reentrant ventricular branch: a method of treating macro-reentrant ventricular tachycardia

27 Wilber DJ, Baerman J, Olshansky B, Kall J, Kopp D. Adenosine-sensitive ventricular tachycardia: clinical characteristics and response to catheter ablation. Circulation 1993;87:126-34.

28 Nakagawa $\mathrm{H}$, Beckman $\mathrm{KJ}$, McClelland JH, Wang X, Arruda M, Santoro I, et al. Radiofrequency catheter ablation of idiopathic left ventricular tachycardia guided by a Purkinje potential. Circulation 1993;88:2607-17.

29 Morady F, Harvey M, Kalbfleisch SJ, el-Atassi R, Calkins $\mathrm{H}$, Langberg JJ. Radiofrequency catheter ablation of ventricular tachycardia in patients with coronary artery disease. Circulation 1993;87:363-72.

30 Wilber DJ, Garan H, Finkelstein D, Kelly E, Newell J, $\mathrm{McG}$ overn $\mathrm{B}$, et al. Out-of-hospital cardiac arrest. Use of McGovern B, et al. Out-of-hospital cardiac arrest. Use of electrophysiologic testing in the prediction
outcome. $N$ Engl f Med 1988;318:19-24.

31 Garratt CJ. Who needs ventricular stimulation studies? $\mathrm{Br}$ Heart f 1994;71:307-8. 